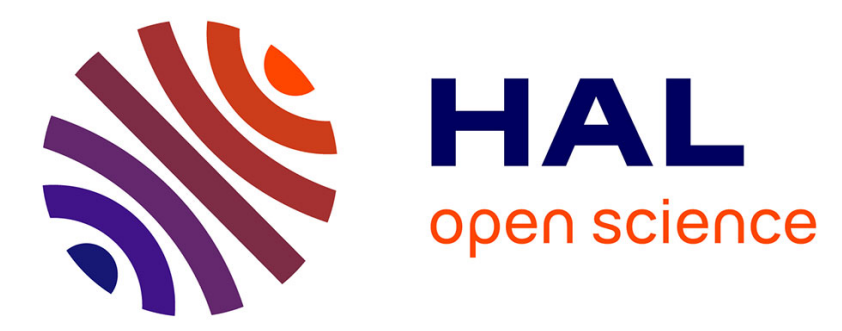

\title{
Cooperativity length scale in nanocomposites: Interfacial and confinement effects
}

Allisson Saiter, D. Prevosto, E. Passaglia, H. Couderc, L. Delbreilh, J. Saiter

\section{To cite this version:}

Allisson Saiter, D. Prevosto, E. Passaglia, H. Couderc, L. Delbreilh, et al.. Cooperativity length scale in nanocomposites: Interfacial and confinement effects. Physical Review E: Statistical, Nonlinear, and Soft Matter Physics, 2013, 88 (4), 10.1103/PhysRevE.88.042605 . hal-02156914

\section{HAL Id: hal-02156914 https://hal.science/hal-02156914}

Submitted on 18 Jun 2019

HAL is a multi-disciplinary open access archive for the deposit and dissemination of scientific research documents, whether they are published or not. The documents may come from teaching and research institutions in France or abroad, or from public or private research centers.
L'archive ouverte pluridisciplinaire HAL, est destinée au dépôt et à la diffusion de documents scientifiques de niveau recherche, publiés ou non, émanant des établissements d'enseignement et de recherche français ou étrangers, des laboratoires publics ou privés. 


\title{
Cooperativity length scale in nanocomposites: Interfacial and confinement effects
}

\author{
A. Saiter, ${ }^{1, *}$ D. Prevosto, ${ }^{2}$ E. Passaglia, ${ }^{3}$ H. Couderc,${ }^{4}$ L. Delbreilh, ${ }^{1}$ and J. M. Saiter ${ }^{1}$ \\ ${ }^{1}$ AMME-LECAP EA4528 International Laboratory, Institut des Matériaux de Rouen, Université et INSA de Rouen, \\ BP12, 76801 Saint Etienne du Rouvray Cedex, France \\ ${ }^{2}$ Institute for Chemical and Physical processes- CNR Largo Pontecorvo 3, 56127 Pisa, Italy \\ ${ }^{3}$ ICCOM-CNR UOS Pisa, Via G. Moruzzi 1, 56124 Pisa, Italy \\ ${ }^{4}$ Institut de Recherche d'HydroQuébec, 1800, boul. Lionel-Boulet, Varennes (Québec) J3X 1S1, Canada
}

(Received 23 January 2013; revised manuscript received 10 September 2013; published 18 October 2013)

\begin{abstract}
Interfacial and confinement effects on the evolution of cooperativity on approaching the glass transition have been studied in poly(propylenecoethylene) functionalized with diethylmaleate, polyethylene 1,4cyclohexylenedimethylene terephthalate glycol and their nanocomposites with montmorillonite. A small increase of the structural dynamic cooperativity, a weak alteration of the temperature dependence of the characteristic relaxation frequency, and no changes in the glass transition temperature observed in poly(propylenecoethylene)based samples can be rationalized in terms of interfacial interactions between polymer and exfoliated clay. On the other hand, confinement of polymer chains in the galleries of clay (intercalated nanocomposite) produces a strong reduction of cooperativity, of the temperature dependence of the characteristic relaxation frequency, and of the glass transition temperature in polyethylene 1,4-cyclohexylenedimethylene terephthalate glycol samples. Finally, by investigating the temperature dependence of a generalized fragility and of cooperativity, we evidenced that fragility of glass formers is determined not only by cooperativity.
\end{abstract}

DOI: 10.1103/PhysRevE.88.042605

PACS number(s): 61.25.H-, 64.70.pj, 77.84.Jd

\section{INTRODUCTION}

Since the early 1990s great attention has been paid to the effects of interfaces and geometrical confinement on dynamics of glass formers [1-14]. It is reported that polymers and simple liquids exhibit drastic structural relaxation modifications near the glass transition when they are geometrically constrained, in comparison to the bulk [15,16], even if some results suggest the opposite [9]. Numerous strategies can be used to produce confinement effects: filling porous glasses with glass formers [17], preparing ultrathin films [18], microemulsions [19], dispersing glass formers into clays [20], and others. Two competing effects are traditionally considered to influence the relaxation dynamics: the reduction of constraints on the development of intermolecular cooperativity and the interactions occurring at the interfaces. Moreover, recently, the idea of the existence of a polymer layer adsorbed at the surface of the confining media was strongly supported by experiments [21-23].

When a glass-forming liquid is cooled down towards the glass transition a dramatic increase of the viscosity and the structural $\alpha$ relaxation times $\tau$ is observed. This phenomenon can be most naturally interpreted assuming a cooperative behavior of the relevant molecular motions. Adam and Gibbs [24] have introduced the notion of the cooperative rearranging region (CRR) that is defined as the smallest amorphous domain where a conformational rearrangement may occur without causing rearrangements in its surrounding.

One distinctive feature of glass formers is the nonArrhenius dependence of the structural relaxation time $\tau$. The deviation from the Arrhenius behavior has been traditionally related to an increase of cooperativity in the relaxation process of interest and it can be quantified by the fragility index $m[25,26]$, defined by $m=\left.\frac{d \log \tau}{d(T g / T)}\right|_{T=T g}$, which is

*Corresponding author: allison.saiter@univ-rouen.fr a material-dependent parameter. Even if the non-Arrhenius behavior of the temperature dependence of relaxation time has been usually ascribed to the cooperativity of the process, establishing a quantitative relationship between the size of the CRR and the fragility is not obvious since many other physical and chemical parameters must be considered, such as density, macromolecular organization [27,28], microstructural organization [29,30], backbone rigidity [31], inter- and intramolecular interactions [32], and mean coordination number in the case of chalcogenide glasses [33]. From the experimental point of view, care should be paid to study the variation of fragility and CRR size by changing few and selected parameters. Moreover, several approaches can be used to estimate fragility [34-37] and the CRR size [38-41], which make the comparisons of different results less clear.

In this paper we want to investigate the effect of confinement and interfacial interaction on relaxation dynamics and cooperativity. In particular, with respect to a previous paper, the novelty is to compare the evolution of relaxation time and cooperativity with temperature, in bulk and confined geometry. We explore if there is any relation between the variation of CRR size and fragility when confinement effects are introduced by dispersing nanoclay in the two polymer matrices. Comparing the pure matrices with the corresponding nanocomposite should highlight the effect of confinement and interfacial interaction, not introducing the effect of other material-dependent physical and chemical parameters.

\section{EXPERIMENT}

A. Sample preparation

Poly(propylenecoethylene) functionalized with diethylmaleate (DEM) (EPMgDEM, functionalization degree = $1.4-1.5 \%$ by mole) was used as matrix for the preparation 
of nanocomposite by mixing with organophilic modified montmorillonite (MMT), containing dimethyl dehydrogenated tallow quaternary ammonium salt as surfactant. The detailed preparation for the samples herein used can be found in Refs. [21,42]. In particular, the pure matrix and the exfoliated structure nanocomposite with nominal content of MMT equal to $5 \%$ by weight (S5) are considered for the present investigation. The composite sample (S5) was completely exfoliated as a result of XRD and TEM analysis [21,42]. Before measurements samples were dried under vacuum at about $308 \mathrm{~K}$ for 1 day.

A second series of polymer-clay nanocomposites was obtained by mixing polyethylene 1,4-cyclohexylenedimethylene terephthalate glycol (PETg) filled and MMT C15A following a master-batch process. Structural characterization of PETg nanocomposite can be found in Ref. [43,44]. In particular, the pure matrix and the nanocomposite with nominal content of MMT equal to $2 \%$ by weight (PETg2\%MMT) with a basal distance of $3.48 \mathrm{~nm}$ are considered for the present investigation. The value of basal distance in nanocomposite has been compared with the basal distance of MMT C15A that is $3.15 \mathrm{~nm}$, thus inferring its intercalated nature. Before measurements, samples were dried overnight in a vacuum oven at $353 \mathrm{~K}$.

\section{B. Experimental techniques}

Dielectric relaxation spectra were measured with an Alpha Analyzer from Novocontrol (frequency interval $10^{-2}-10^{7} \mathrm{~Hz}$ ). A film of the material under study was placed in a parallel plate capacitor and the temperature was controlled through a heated flow of nitrogen gas by means of a Quatro Cryosystem in the interval 143-273 $\mathrm{K}$ for the EPMgDEM sample and its nanocomposite, and in the interval 193-433 K for PETg sample and its nanocomposite. During the whole period of the measurement the sample was kept in a pure nitrogen atmosphere.

Temperature-modulated differential scanning calorimetry (TMDSC) experiments are performed in a Thermal Analysis instrument (TA Q100) with an oscillation amplitude of $1 \mathrm{~K}$, an oscillation period of $60 \mathrm{~s}$, and with a cooling rate of $0.5 \mathrm{~K} / \mathrm{min}$ from the liquidlike state. Calibration in temperature and energy is carried out using standard values of indium and zinc, and the specic heat capacity for each sample is measured using sapphire as a reference. The details about calibration are given in Ref. [45]. The sample masses are chosen to be similar to the sapphire sample mass, i.e., approximately $20 \mathrm{mg}$. Before TMDSC experiments, all the samples have been dried under vacuum sustain a thermal treatment in order to avoid thermal history effects.

\section{RESULTS}

The detailed analysis of isothermal dielectric spectra of EPMgDEM and its nanocomposite S5 have been presented in a previous publication, to which the interested reader can refer [46]. In the present paper some results of the previous analysis has been considered, together to new TMDSC measurements.

In Fig. 1(a) isofrequency spectra of imaginary part of dielectric function, $\varepsilon^{\prime \prime}$, as a function of the temperature are
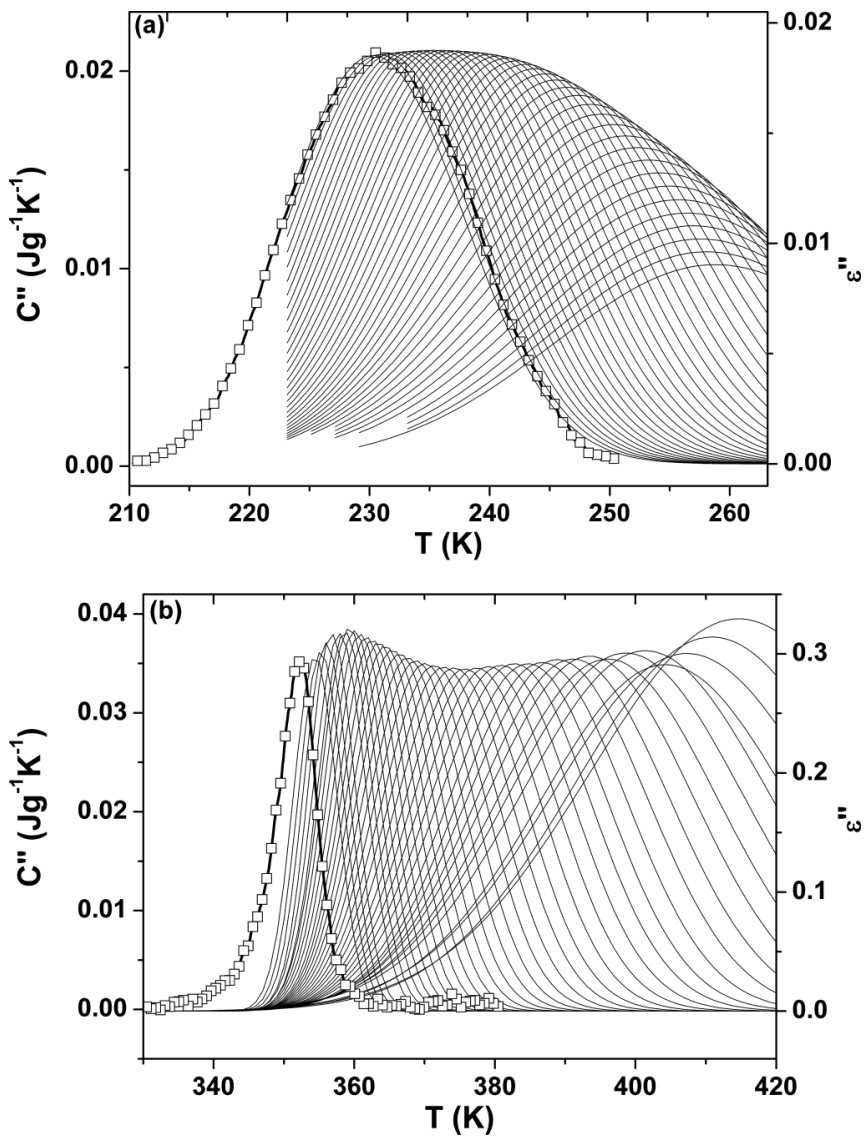

FIG. 1. (a) Dielectric loss isofrequency spectra of pure EPMgDEM, $\varepsilon^{\prime \prime}(T)$ [thin lines for frequencies from $5 \times 10^{-2} \mathrm{~Hz}$ to $4 \times 10^{5}$ Hz with $\Delta \log _{10}(\omega)=0.16$ between two successive curves] and $C^{\prime \prime}(T)$ curve (line with squares for the frequency of $1.6 \times 10^{-2} \mathrm{~Hz}$ ). (b) Dielectric loss isofrequency spectra of pure PETg, $\varepsilon^{\prime \prime}(T)$ [thin lines for frequencies from $9 \times 10^{-1} \mathrm{~Hz}$ to $4 \times 10^{6} \mathrm{~Hz}$ with $\Delta \log _{10}(\omega)=$ 0.16 between two successive curves] and $C^{\prime \prime}(T)$ curve (line with squares for the frequency of $1.6 \times 10^{-2} \mathrm{~Hz}$ ).

plotted for frequencies from $5 \times 10^{-2} \mathrm{~Hz}$ to $4 \times 10^{5} \mathrm{~Hz}$ for EPMgDEM. The same plots obtained for PETg are shown in Fig. 1(b) for frequencies from $9 \times 10^{-1} \mathrm{~Hz}$ to $4 \times 10^{6} \mathrm{~Hz}$. In both cases, a peak corresponding to the structural or $\alpha$ relaxation process can be observed. Such spectra are plotted by subtracting the contribution from the conductivity and the secondary relaxation, which have been estimated in the previous analyses showed in Ref. [46] for EPMgDEM and its nanocomposite, and in Ref. [44] for PETg and its nanocomposite. For comparison, in the same figure one spectrum of the out-of-phase contribution of the specific heat capacity obtained by TMDSC, $\mathrm{C}^{\prime \prime}$, is reported for the frequency of $1.6 \times 10^{-2} \mathrm{~Hz}$.

It can be observed that the broadness of the $\varepsilon^{\prime \prime}$ and $\mathrm{C}^{\prime \prime}$ peaks are comparable, that is, the frequency distribution of the contributions to the structural dielectric and calorimetric relaxation match quite nicely. This result agrees with previous observation on polybisphenol A carbonate [47], as well as investigation on glycerol [48-50]. The temperature curves of $\varepsilon^{\prime \prime}$ and $\mathrm{C}^{\prime \prime}$ are fitted to a Gaussian function in order to obtain the parameters $T_{\alpha}$ and $\delta T$ necessary to calculate the CRR size 
according to the approach proposed by Donth [51],

$$
V_{\alpha}=\frac{\Delta\left(C_{\mathrm{v}}^{-1}\right)}{\rho(\delta T)^{2}} k_{\mathrm{B}} T_{\alpha}^{2},
$$

where a difference in reciprocal specific heat capacity $\Delta\left(C_{\mathrm{v}}{ }^{-1}\right)$ is determined at $T_{\alpha}$ from the in-phase component of the specific heat capacity $\mathrm{C}^{\prime}$, assuming that $C_{\mathrm{v}}$ values are close to $C_{\mathrm{p}}$ values,

$$
\Delta\left(C_{\mathrm{v}}^{-1}\right) \approx \Delta\left(C_{\mathrm{p}}^{-1}\right)=\left(C_{\mathrm{p}}^{-1}\right)_{\text {glass }}-\left(C_{\mathrm{p}}^{-1}\right)_{\text {liquid }} .
$$

$\delta T$ is the mean temperature fluctuation related to the dynamic glass transition of an average CRR and $\rho$ is the glass density. The number of monomer units per CRR noted $N_{\alpha}$ can be estimated as

$$
N_{\alpha}=\frac{\rho N_{\mathrm{A}} V_{\alpha}}{M_{0}}=\frac{N_{\mathrm{A}} \Delta\left(C_{\mathrm{p}}^{-1}\right)}{M_{0}(\delta T)^{2}} k_{\mathrm{B}} T_{\alpha}^{2},
$$

where $N_{\mathrm{A}}$ is the Avogadro number and $M_{0}$ the molar mass of one monomer unit $\left(218 \mathrm{~g} \mathrm{~mol}^{-1}\right.$ for PETg and its nanocomposite and $33 \mathrm{~g} \mathrm{~mol}^{-1}$ for EPMgDEM and its nanocomposite).

Looking in details to the temperature dependence of the relaxation frequency corresponding to the dielectric $\alpha$ peak, $\nu^{\alpha}{ }_{\max }$, we can observe a small but systematic shift to higher values for S5 respect to pure EPMgDEM [Fig. 2(a)]. Such a shift is not constant with temperature and tends to decrease on approaching the dynamic glass transition temperature, $T_{g}$ [inset of Fig. 2(a)], defined as $v^{\alpha}{ }_{\text {max }}=(20 \pi)^{-1}$ (or, equivalently, $\tau^{\alpha}{ }_{\max }=10 \mathrm{~s}$ ). The choice of reference value of $\tau^{\alpha}{ }_{\text {max }}=10 \mathrm{~s}$ for $T_{g}$ has been done to avoid the extrapolation of $\tau^{\alpha}{ }_{\text {max }}(T)$ curves over more one decade in time, which can introduce large errors with respect to the small variations we want to observe. Such a choice slightly differs from the traditional one but is sometimes used [52,53]. Such a scenario quantitatively is reflected in a fragility parameter $m=99 \pm$ 4 and $T_{g}=228.3 \pm 3 \mathrm{~K}$ for S5 and $m=91 \pm 3$ and $T_{g}=$ $227.2 \pm 3 \mathrm{~K}$ for the pure polymer (Table I).

Though the $T_{g}$ values are consistent within the errors, the difference in fragility is substantial. Moreover, looking at the ratio of the relaxation frequencies of the two samples measured at the same temperature [inset in Fig. 2(a)], the presence of different temperature dependencies of $v^{\alpha}{ }_{\text {max }}(T)$ can be affirmed without doubt. In parallel, the same plots are shown in Fig. 2(b) for pure PETg and PETg2\%MMT. The tendency clearly differs: The shift tends to increase on approaching $T_{g}$ [inset in Fig. 2(b)], reflecting the minor fragility of the nanocomposite with respect to the pure material. The origin of such a difference should be related to the different arrangement of the polymer. Indeed, in one case the nanocomposite has an exfoliated structure and in the other an intercalated one, in which the polymer chains that have been introduced between clay platelets are confined in a restricted region of space because of the small basal distance.

However, the amount of molecules affected by the confinement effect seems to be much larger than just the intercalated chains and is probably at the origin of the observed speeding up of dynamics and reduction of dynamic glass transition
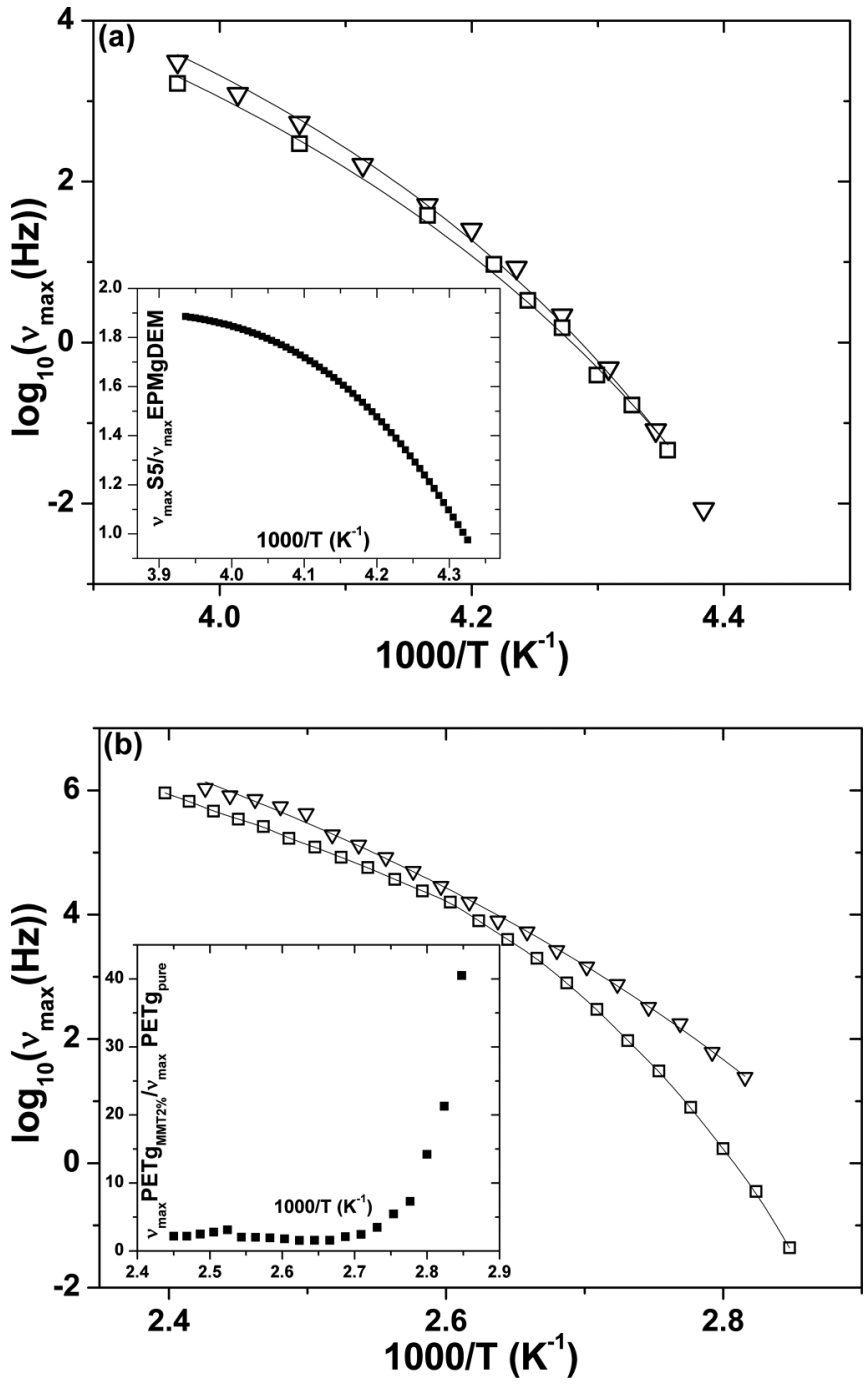

FIG. 2. (a) Arrhenius plot of EPMgDEM (squares) and S5 (triangles) samples together with VFT fit (lines). In the inset the ratio between relaxation frequency of S5 and EPMgDEM is reported as a function of inverse temperature. (b) Arrhenius plot of PETg (squares) and with 2\% MMT (triangles) samples together with VFT fit (lines). In the inset the ratio between relaxation frequency of PETg2\%MMT and pure PETg is reported as a function of inverse temperature.

temperature of the nanocomposite PETg2\%MMT with respect to the pure PETg (see Table I) $[43,54]$. In the following we will investigate the origin of such results in terms of the CRR size temperature evolution.

TABLE I. Glass transition temperature $\left(T_{g}\right)$ and fragility index $(m)$ of EPMgDEM and PETg, pure polymer, and corresponding nanocomposite.

\begin{tabular}{lrrrrr}
\hline \hline & \multicolumn{2}{c}{ EPMgDEM } & & \multicolumn{2}{c}{ PETg } \\
\cline { 2 - 3 } \cline { 5 - 6 } & \multicolumn{1}{c}{ Pure } & 5\% MMT & & Pure & $2 \%$ MMT \\
\hline$T_{g}(\mathrm{~K})$ & $227.2 \pm 3$ & $228.3 \pm 3$ & & $350 \pm 3$ & $336 \pm 3$ \\
$m$ & $91 \pm 3$ & $99 \pm 4$ & & $125 \pm 5$ & $71 \pm 3$ \\
\hline
\end{tabular}



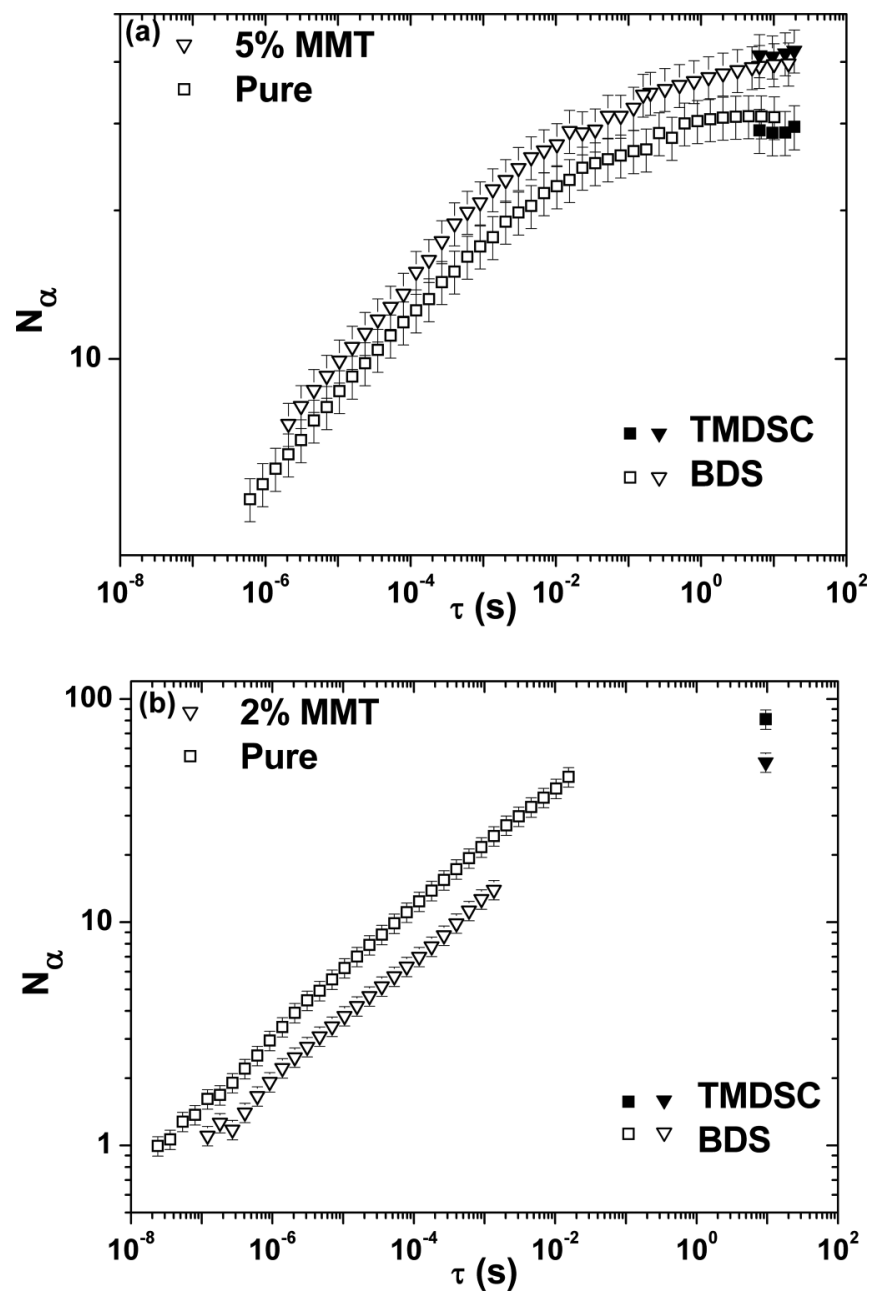

FIG. 3. (a) Evolution of $N_{\alpha}$ versus relaxation time for EPMgDEM (triangles) and S5 (squares). $N_{\alpha}$ is calculated from TMDSC experiments (filled squares and triangles) and BDS experiments (hollow squares and triangles). (b) Evolution of $N_{\alpha}$ versus relaxation time for pure PETg (squares) and PETg2\%MMT (triangles). $N_{\alpha}$ is calculated from TMDSC experiments (filled squares and triangles) and BDS experiments (hollow squares and triangles).

\section{DISCUSSION}

In this work, we choose to use the model proposed by Donth [51] and its recent extension [47] to calculate the size of the CRR $V_{\alpha}$, or the number of monomers comprised in a cooperatively rearranging region, $N_{\alpha}$. The use of the extended Donth model [47] allows us to investigate the variation of $N_{\alpha}$ in broad relaxation time or temperature intervals.

In Fig. 3(a) $N_{\alpha}$ is presented as a function of the relaxation time for EPMgDEM samples. Here the relaxation time is $\tau=(2 \pi \nu)^{-1}$, where $\nu$ is the frequency of the isochronal spectrum of $\varepsilon^{\prime \prime}$ or $C^{\prime \prime}$ used to estimate the parameters of Eq. (3) $N_{\alpha}$ increases on approaching the glass transition (here represented at $\tau=10 \mathrm{~s}$ ), in agreement with the idea that the cooperativity of glass formers increases as it approaches $T_{g}$. We notice that $N_{\alpha}$ values from dielectric spectroscopy and TMDSC measurements agree with each other close to $T_{g}$. These results confirm those previously found in polybisphenol
A carbonate [47]. Comparing the effect of clay inclusion on the CRR size in the two nanocomposites we find differences. In the case of well-exfoliated S5, $N_{\alpha}$ is systematically slightly larger than in pure EPMgDEM, whereas in the case of intercalated PETg2\%MMT $N_{\alpha}$ is smaller compared to the pure PETg [Fig.3(b)]. A decrease of $N_{\alpha}$ (or $V_{\alpha}$ ) with confinement has been already reported in the literature $[55,56]$.

On the other hand, the increase of $N_{\alpha}$ in S5 nanocomposite can be explained in terms of favorable interactions between the platelets and the polymer chains [21,46]. In fact, because of such positive interactions the platelets are surrounded by a region of polymer chains that behaves as bound to the clay, where likely a larger cooperativity is present with respect to the pure polymer. The presence of such a region of bound polymer has been evidenced through an extraction procedure showing the presence of a residue constituted by the clay and the polymer itself [21]. Such a residue is no more soluble in the polymer solvent and can be considered strictly interacting with the clay. A similar phenomenon, i.e., the adsorption of polymer chain at the interface with an inorganic component, has been also evidenced to occur at the polymer-metal surface in the polymer film $[22,23,57]$. Moreover, a higher-density region of polymer close to MMT has been found in simulation [58].

Also the temperature dependence of the relaxation time is affected by confinement. In particular, it decreases with confinement in analogy to the CRR size. In fact, S5 has larger fragility than EPMgDEM and also a larger value of $N_{\alpha}$ at the glass transition. Moreover, the temperature dependence of $v^{\alpha}{ }_{\max }$ is stronger and stronger for S5 with respect to EPMgDEM at $T$ decrease towards $T_{g}$ [inset Fig. 2(a)] and, correspondingly, we have in the same $T$ region a larger deviation of $N_{\alpha}$ between the two systems [Fig. 3(a)]. On the other hand, in PETg samples a reduction of fragility from 121 to 71 is obtained in the nanocomposite, as well as a reduction of the number of $N_{\alpha}$. Interestingly, such results agree with previous investigations in pure materials, evidencing a rough relation between fragility and cooperativity $[38,39,59,60]$ when other molecular properties are maintained fixed, even though in the previous papers the estimation of the cooperativity has been performed according to a different model with respect to the one used here. It is important to mention that the relationship between any parameter and fragility should be searched among materials with no large differences in molecular structure, as pointed out in an analysis of a vast database of experimental results [53].

Regarding the increase of $N_{\alpha}$ on approaching the glass transition, for EPMgDEM and its nanocomposite the dependence on the relaxation time can be reproduced by two power-law regimes. A first one occurs for relaxation times smaller than about $10^{-2} \mathrm{~s}$ (far from $T_{g}$ ), where the increase of $N_{\alpha}$ is steeper than the increase in the second region. In particular, it seems that $N_{\alpha}$ tends to a constant limit value at the glass transition $[47,61]$. This behavior contrasts with the common idea that the cooperativity of a glass former should diverge on approaching the glass transition. However, we found this result to be quite general. In fact, the $N_{\alpha}$ values of PETg samples (lower panel in Fig. 3) are present at the glass transition (evaluated from TMDSC) and in the region for $\tau<10^{-2}-10^{-3} \mathrm{~s}$. In the short time region a single power law is sufficient to describe the increase of $N_{\alpha}$ with $\tau$. However, if we want to reconcile the 

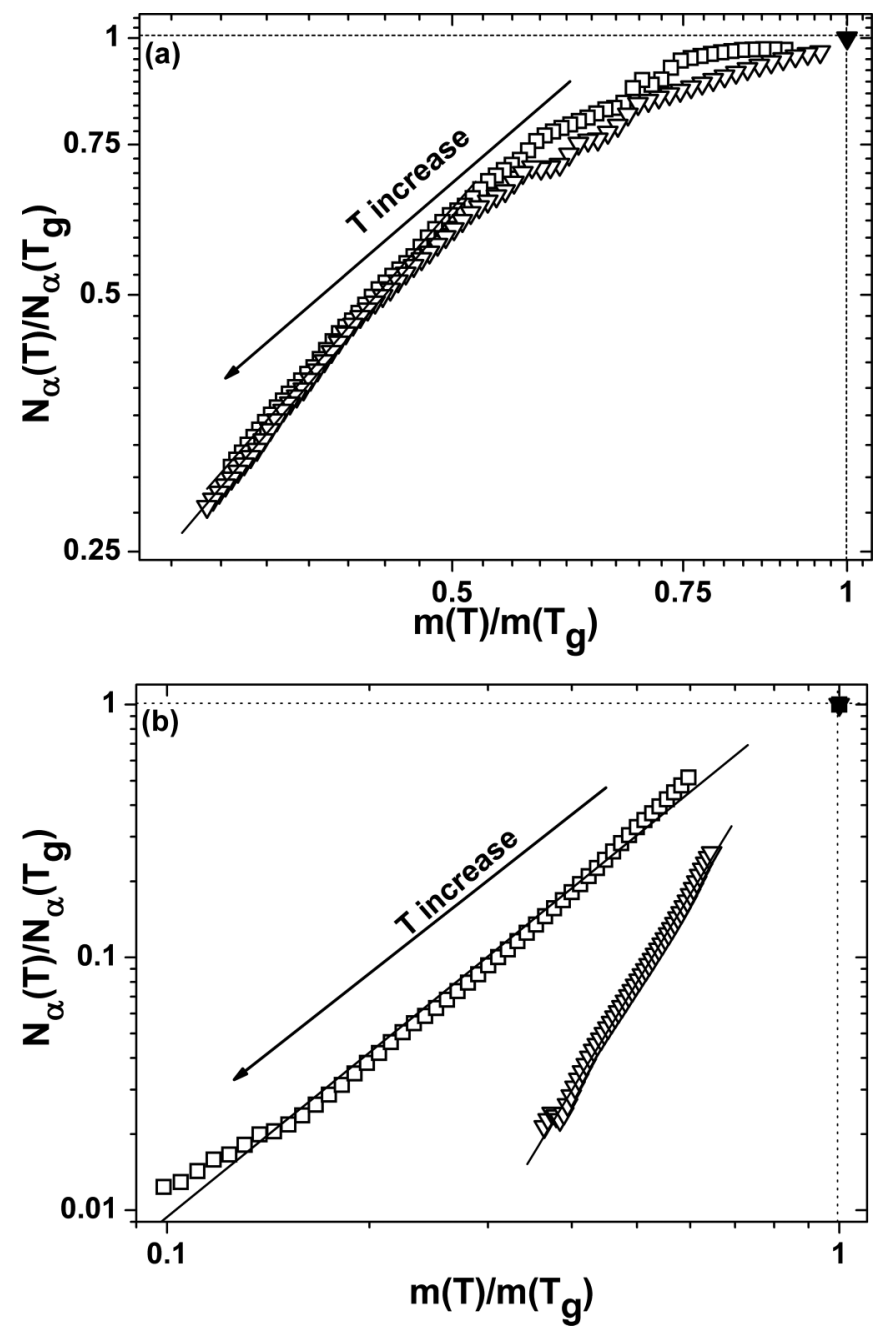

FIG. 4. (a) Evolution of $N_{\alpha}$ versus fragility; both values are normalized to their values at $T_{g}$ for pure EPMgDEM (squares) and S5 (triangles). (b) Evolution of $N_{\alpha}$ versus fragility, both values are normalized to their values at $T_{g}$, for pure PETg (squares) and PETg2\%MMT (triangles). Power-law adjustments $N_{\alpha}=A m^{B}$ (lines).

high temperature values of $N_{\alpha}$ from dielectric experiment to the low temperature one from TMDSC, we have to hypothesize a transition to a less step power law for the PETg samples. Then the same trend can be reasonably suggested for all the four samples herein investigated. This trend has also been clearly observed for polycarbonate [47] with a $N_{\alpha}$ steep evolution for small relaxation times, and an important slow-down of this variation for $\tau>10^{-3} \mathrm{~s}$.

Finally, it is interesting to compare the $T$ dependence of $N_{\alpha}$ and the parameter

$$
m(T)=d \log \tau / d\left(\frac{T_{g}}{T}\right),
$$

which, for $T=T_{g}$, is the usual fragility [35].

In Fig. 4(a), $N_{\alpha}$ is plotted for EPMgDEM and S5 samples as a function of $m$ rescaled for values at $T_{g}$. We can observe that $N_{\alpha}$ is levels out on approaching the glass transition. For PETg and its nanocomposite we have data only far from $T_{g}$. In the region of investigation we observe that a power-law relation exists between them, i.e., $N_{\alpha}=A m^{B}$, with both $A$ and $B$ material-dependent parameters [lines in Fig. 4(b)]. However, if we want to reconcile the high temperature data of PETg samples with the data at $T_{g}$, it seems that a deviation from the above-mentioned power law should be considered, analogously with the case of polyolefin samples. We can conclude that close to the glass transition the parameter $m$ increases faster than $N_{\alpha}$; consequently, it appears that factors other than cooperativity participate in determining the non-Arrhenius behavior of characteristic time on approaching $T_{g}$.

We make a final remark on the power law describing $N_{\alpha}(\tau)$ curves at short value of $\tau$. In particular, $B$ is equal to 2.2 for PETg and 4.4 for its nanocomposite. Interestingly, if we analyze the data of polyolefin samples at low values of $m$ $\left[m<0.5 m\left(T_{g}\right)\right]$ a single power law with exponent $B=1.8$ can be used for both samples. In particular, due to the scattering of the data, the exponent $B$ for PETg and the two polyolefins can be considered equal.

\section{CONCLUSIONS}

The evolution of cooperativity and relaxation time on approaching the glass transition has been studied in two pure polymers, i.e., poly(propylenecoethylene) functionalized with diethylmaleate, EPMgDEM, and polyethylene 1,4cyclohexylenedimethylene terephthalate glycol, PETg, and their nanocomposites with montmorillonite. Interfacial interactions dominate in the EPMgDEM nanocomposite, which has an exfoliated morphology, whereas confinement effects are more important in the PETg nanocomposite, which has an intercalated morphology. We retain this hypothesis on the role of interfacial interactions and confinement to give a consistent interpretation of the observed properties. In fact, we explain accordingly that interfacial interactions produce a weak alteration of the relaxation and cooperativity properties on the examined nanocomposite, the main results being a slight increase of cooperativity regions. Such an increase could be due to adsorption of a polymer chain around MMT platelets, producing a more constrained and dense region of polymers in the material. In intercalated nanocomposites larger deviations with respect to the polymers are apparent, as a reduction of $T_{g}$ and a strong reduction of cooperativity regions. Such a reduction could be due to the restriction imposed by the MMT platelets on the polymer chain intercalated between them. Moreover, our study permitted us to investigate the relation between the temperature dependence of the structural relaxation frequency and the cooperativity of the materials. It emerges that these two properties follow a similar trend when considering chemically similar materials. In fact, a reduction of $N_{\alpha}$ is accompanied by a reduction of fragility and vice versa. However, it also comes out that on approaching the glass transition the number of cooperative units (or correlated segments) seems to level off to a constant value, whereas the slope of the temperature dependence of the relaxation frequency increases faster and faster. This observation evidences that the fragility of a glass former material is not only related to the number of units in the cooperative region.

We stress that a quantitative relation between the amount of the affected polymer and the observed change of property is not achieved, as it would require a reliable model quantitatively 
relating microscopic properties of the material (number of units in a cooperative region, intensity of interfacial interaction and others) that, as far as we know, is still missing. For this reason, our discussion of the results, as many others in the field, is mainly on a qualitative level.

\section{ACKNOWLEDGMENTS}

D.P. acknowledges University of Rouen for support during a visit. M.B. at IPCF-CNR is thanked for the help in sample preparation and discussion.
[1] J. M. Drake, G. M. Great, and J. Klafter, in Dynamics in Small Confining Systems IV, edited by R. Kopelman (Materials Research Society, Pittsburgh, 1999).

[2] Y. Braiman, J. M. Drake, and F. Family, in Dynamics and Friction in Submicrometer Confining Systems, ACS Symposium Series, Vol. 882, edited by J. Klafter (ACS Books, Washington, DC, 2004).

[3] C. L. Jackson and G. B. McKenna, J. Non-Cryst. Solids 131-133, 221 (1991).

[4] C. Schick, Eur. Phys J. Spec. Topics 189, 3 (2010).

[5] R. Richert, Eur. Phys J. Spec. Topics 189, 37 (2010).

[6] K. Fukao, Y. Od, K. Nakamura, and D. Tahara, Eur. Phys J. Spec. Top. 189, 165 (2010).

[7] S. Napolitano and M. Wubbenhorst Polymer 51, 5309 (2010).

[8] M. Erber, A. Khalyavina, K. J. Eichhorn, and B. I. Voit, Polymer 51,129 (2010).

[9] E. U. Mapesa, M. Erber, M. Tress, K. J. Eichhorn, A. Serghei, B. Voit, and F. Kremer, Eur. Phys J. Spec. Top. 189, 173 (2010).

[10] M. Erber, U. Georgi, J. Muller, K. J. Eichhorn, and B. Voit, Eur. Pol. J. 46, 2240 (2010).

[11] A. Saiter, N. Delpouve, E. Dargent, and J. M. Saiter, Eur. Pol. J. 43, 4675 (2007).

[12] C. Lixon, N. Delpouve, A. Saiter, E. Dargent, and Y. Grohens, Eur. Pol. J. 44, 3377 (2008).

[13] N. Delpouve, A. Saiter, and E. Dargent, Eur. Pol. J. 47, 2414 (2011).

[14] H. Couderc, A. Saiter, J. Grenet, and J. M. Saiter, Physica B 406, 2908 (2011).

[15] M. D. Ediger, C. A. Angell, and S. R. Nagel, J. Phys. Chem. 100, 13200 (1996).

[16] G. B. McKenna, J. Phys. IV 10, 53 (2000).

[17] J. Zhang, G. Liu, and J. Jonas, J. Phys. Chem. 96, 3478 (1992).

[18] J .K. Keddie, R. A. L. Jones, and R. A. Cory, Eur. Lett. 27, 59 (1994).

[19] L. M. Wang, F. He, and R. Richert, Phys. Rev. Lett. 92, 095701 (2004).

[20] R. Bergman, J. Swenson, L. Borjesson, and P. Jacobsson, J. Chem. Phys. 113, 357 (2000).

[21] E. Passaglia, M. Bertoldo, F. Ciardelli, D. Prevosto, and M. Lucchesi, Eur. Pol. J. 44, 1296 (2008).

[22] Y. Fujii, Z. Yang, J. Leach, H. Atarashi, K. Tanaka, and O. K. C. Tsui, Macromolecules 42, 7418 (2009).

[23] S. Napolitano and M. Wübbenhorst, Nat. Commun. 2, 260 (2011).

[24] G. Adam and J. H. Gibbs, J. Chem. Phys. 43139 (1965).

[25] C. A. Angell, J. Non-Cryst. Solids 131-133, 13 (1991).

[26] D. J. Plazek and K. L. Ngai, Macromolecules 24, 1222 (1991).

[27] A. Saiter, C. Devallencourt, J. M. Saiter, and J. Grenet, Eur. Polym. J. 37, 1083 (2001).

[28] A. Saiter, M. Hess, N. A. D'Souza, and J. M. Saiter, Polymer 43, 7497 (2002).
[29] R. Crétois, L. Delbreilh, E. Dargent, N. Follain, L. Lebrun, and J.-M. Saiter, Eur. Polym. J. 49, 3434 (2013).

[30] F. Hamonic, A. Saiter, D. Prevosto, E. Dargent, and J. M. Saiter, in 6th International Conference on Times of Polymers (TOP) and Composites, Ischia, Italy, AIP Conf. Proc. No. 1459 (AIP, New York, 2012), p. 211.

[31] L. Delbreilh, A. Bernes, C. Lacabanne, J. Grenet, and J. M. Saiter, Mater. Lett. 59, 2881 (2005).

[32] N. Delpouve, A. Vuillequez, A. Saiter, B. Youssef, and J. M. Saiter, Physica B 407, 3561 (2012).

[33] G. Saffarini, A. Saiter, M. R. Garda, and J. M. Saiter, Physica B 389, 275 (2007).

[34] L. M. Wang, V. Velikov, and C. A. Angell, J. Chem. Phys. 117, 10184 (2002).

[35] K. Niss, C. Dalle-Ferrier, G. Tarjus, and C. Alba-Simionesco, J. Phys.: Condens. Matter 19, 076102 (2007).

[36] J. M. Saiter, J. Grenet, E. Dargent, A. Saiter, and L. Delbreilh, Macromol. Symp. 258, 152 (2007).

[37] L. Delbreilh, M. Negahban, M. Benzohra, C. Lacabanne, and J. M. Saiter, J. Thermal Analy. Calorimetry 96, 865 (2009).

[38] A. Saiter, H. Couderc, and J. Grenet, J. Therm. Anal. Calorim. 88, 483 (2007).

[39] A. Saiter, J. M. Saiter, and J. Grenet, Eur. Pol. J.42, 213 (2006).

[40] L. Hong, P. D. Gujrati, V. N. Novikov, and A. P. Sokolov, J. Chem. Phys. 131, 194511 (2009).

[41] C. M. Roland, D. Fragiadakis, D. Coslovich, S. Capaccioli, and K. L. Ngai, J. Chem. Phys. 133, 124507 (2010).

[42] E. Passaglia, R. Sulcis, F. Ciardelli, M. Malvaldi, and P. Narducci, Polymer Int. 54, 1549 (2005).

[43] H. Couderc, L. Delbreilh, A. Saiter, J. Grenet, N. De Souza, and J. M. Saiter, J. Non-Cryst. Solids 353, 4334 (2007).

[44] H. Couderc, A. Saiter, J. Grenet, J. M. Saiter, G. Boiteux, E. Nikaj, I. Stevenson, and N. D’Souza, Pol. Eng. Sci. 49, 836 (2009).

[45] C. Schick, in Hand-book of Thermal Analysis and Calorimetry, edited by S. Cheng (Elsevier, New York, 2002). Vol. 3, p. 713.

[46] D. Prevosto, M. Lucchesi, M. Bertoldo, E. Passaglia, F. Ciardelli, and P. A. Rolla, J. Non-Cryst. Solids 356, 568 (2010).

[47] A. Saiter, L. Delbreilh, H. Couderc, K. Arabeche, A. Schonhals, and J. M. Saiter, Phys. Rev. E 81, 041805 (2010).

[48] S. Weinstein and R. Richert, J. Chem. Phys. 123, 224506 (2005).

[49] R. Casalini and C. M. Roland, J. Chem. Phys. 135, 094502 (2011).

[50] L. Hong, V. N. Novikov, and A. P. Sokolov, Phys. Rev. E 83, 061508 (2011).

[51] E. Donth, in The Glass Transition Relaxation Dynamics in Liquids and Disordered Materials, 1st ed. (Springer, Berlin, 2001).

[52] R. Casalini and C. M. Roland, Phys. Rev. B 71, 014210 (2005).

[53] K. L. Ngai in Relaxation and Diffusion in Complex Systems (Springer, New York, 2011). 
[54] S. H. Anastasiadis, K. Karatasos, G. Vlachos, E. Manias, and E. P. Giannelis, Phys. Rev. Lett. 84, 915 (2000).

[55] T. A. Tran, S. Said, and Y. Grohens, Macromolecules 38, 3867 (2005).

[56] K. Arabeche, L. Delbreilh, R. Adhikari, G. H. Michler, A. Hiltner, E. Baer, and J. M. Saiter, Polymer 53, 1355 (2012).

[57] H. K. Nguyen, M. Labardi, S. Capaccioli, M. Lucchesi, P. A. Rolla, and D. Prevosto, Macromolecules 45, 2138 (2012).
[58] R. Toth, D. J. Voorn, J. W. Handgraaf, J. G. E. M. Fraaije, M. Fermeglia, S. Pricl, and P. Posocco, Macromolecules 42, 8260 (2009).

[59] A. Saiter, E. Dargent, J. M. Saiter, and J. Grenet, J. Non-Cryst. Solids 354, 345 (2008).

[60] A. Saiter, E. Bureau, H. Zapolsky, and J. M. Saiter, J. Non-Cryst. Solids 345, 556 (2004).

[61] S. Capaccioli, G. Ruocco, and F. Zamponi, J. Phys. Chem. B 112, 10652 (2008). 